\title{
L'homme et son environnement, ou de la complexité dans le domaine de la santé
}

\section{Pierre Corvol} P. Corvol : Inserm U. 36, Collège de France,
3, rue d'Ulm, 75005 Paris, France.

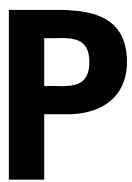
arallèlement aux recherches moléculaires effectuées sur le vivant, les études sur les paramètres physiques, chimiques, biologiques et socio-économiques susceptibles d'agir sur le fonctionnement des organismes vivants et d'influencer le comportement humain se sont développées au cours de la deuxième moitié du $X X^{e}$ siècle. La notion d'environnement, dans son acception actuelle, est récente. La British Ecological Society, la première société d'écologie, a été fondée en 1913 par un petit groupe de botanistes [1, 2]. Au milieu de ce siècle, la société dans son ensemble a pris conscience de l'importance de l'étude de l'environnement. Cet intérêt, cette exigence même, s'exprime par une demande forte, particulièrement médiatisée, de connaissance sur les conséquences des modifications du climat, de I'hydrosphère, de l'alimentation sur la santé de l'homme. Pour le grand public, I'homme a changé les conditions de son milieu, souvent idéalisé (I'air et l'eau pures, la nourriture "biologique», les médecines «douces»), aux dépens d'un univers pollué par les fumées, I'alimentation industrielle et le profit. La société réclame un contrôle de l'environnement, un risque zéro et zéro défaut. La réponse politique s'est traduite dans la plupart des pays par la création de ministères de l'environnement et d'agences sanitaires dont la mission est d'assurer une protection des individus vis-à-vis des risques induits par les activités humaines.

Or sur le plan scientifique, l'étude de I'homme dans son environnement se heurte à un certain nombre de difficultés inhérentes à la connaissance et à la mesure des très nombreux facteurs géographiques, mais aussi sociaux, culturels et économiques dans lesquels évoluent I'homme et la société. A titre d'exemple, des expériences de comportement effectuées dans trois laboratoires différents, menées chez des souris, rigoureusement identiques sur le plan génétique, et dans des conditions standardisées, montrent des différences notables sans que I'on puisse identifier le ou les paramètres environnementaux responsables de ces différences [3].

Un autre élément à prendre en compte est l'évolution des paramètres environnementaux dans le temps et dans l'espace: les changements rapides de l'environnement dus à I'industrialisation et à l'urbanisation, à l'accroissement démographique et aux mouvements de population, impliquent une répétition des mesures et des études épidémiologiques longitudinales de grande ampleur. Une vision à long terme est indispensable: une modification du milieu par l'homme peut n'avoir qu'une répercussion lointaine sur la santé humaine. Un exemple démonstratif est celui de l'épidémie d'encéphalopathie spongiforme bovine dans laquelle l'apparition des premiers signes neurologiques ne survient qu'après un temps de latence de dix ans. Il est difficile de prédire dans ces conditions l'incidence future de la maladie humaine correspondante. Un élément de réponse est la modélisation des événements à venir à partir des données recueillies, permettant leur extrapolation aux populations. Ainsi il est possible de prévoir le nombre approximatif de cas de maladie de Creutzfeldt-Jakob qui pourrait atteindre la Grande-Bretagne dans les dix ans [4].

La vérification expérimentale des données issues de l'observation épidémiologique chez I'homme est difficile. II est possible d'étudier les modifications physiologiques induites chez l'homme sain par des perturbations expérimentales aiguës (conditions physiques, effet des nutriments, des médicaments). Ainsi peut-on apprécier la réponse cardiovasculaire à des conditions extrêmes de température, de pesanteur, d'hydratation. En revanche, il n'est pas possible d'expérimenter en chronique chez l'homme sain et les modèles expérimentaux chez l'animal ne sont que partiellement extrapolables aux conditions humaines.

L'effet des pollutions majeures sur la santé est le plus simple à mettre en évidence: pollution par les métaux 
lourds (mercure, plomb), par les radiations ionisantes (Tchernobyl), par la dioxine... Encore a-t-il fallu attendre la fin du XIX siècle pour que la néphropathie saturnique soit rapportée à l'agent causal. Bien plus délicates sont l'identification et la connaissance du rôle de certains toxiques et polluants chroniques qui agissent à bas bruit. La controverse persistera sur l'influence de la pollution urbaine (la teneur en monoxyde de carbone, les particules de diésel...) sur l'incidence des pneumopathies (alvéolites, asthme...) tant que des études épidémiologiques menées à large échelle, à long terme, et avec la rigueur nécessaire n'auront pas été menées. L'exemple du tabagisme est illustratif à cet égard. Fumer provoque une maladie cardiovasculaire ou un cancer après de longues années et il a fallu les résultats d'une étude épidémiologique effectuée auprès des médecins britanniques en 1964 pour démontrer le lien entre cancer pulmonaire et tabac, alors même que ce dernier a été introduit en Europe au $\mathrm{XV} l^{\mathrm{e}}$ siècle [5]. La preuve du rôle nocif du tabagisme dans les maladies cardiovasculaires fut apportée encore plus tard.

Les changements de comportement alimentaire sont vraisemblablement à l'origine des modifications rapides des indices de masse corporelle et de I'augmentation de l'incidence de I'obésité chez l'enfant et l'adulte, constatées dans tous les pays occidentalisés, encore que d'autres facteurs peuvent en rendre compte (diminution de l'activité physique, accroissement du stress, acculturation de certaines populations). Les liens précis reliant morbi-mortalité et habitudes alimentaires sont donc délicats à établir. Le fameux paradoxe français - une protection relative de notre population vis-à-vis des événements cardiovasculaires, en regard des facteurs de risque similaires à ceux de nos voisins (cholestérolémie, pression artérielle...) - ne serait pas lié à une consommation hexagonale et bénéfique de vin, n'en déplaise aux viticulteurs!

Une voie de recherche qui intéresse au premier chef chaque individu est de connaître la part des facteurs indi- viduels, et notamment génétiques, dans la réponse spécifique à une modification environnementale. De très nombreuses affections dépendent à la fois de facteurs génétiques et environnementaux. Ces derniers prévalent dans le déterminisme du diabète non insulino-dépendant, de I'hypertension artérielle, de l'obésité. Le poids de la génétique dans la variance de la pression artérielle est de $30 \%$, celui de l'environnement de $50 \%$. La complexité tient au fait qu'il existe des interrelations entre plusieurs facteurs génétiques, et entre facteurs génétiques et environnementaux. Le tabagisme ne provoque pas inévitablement un cancer ou un infarctus. Les variations d'ordre génétique du métabolisme des dérivés toxiques de la fumée du tabac commencent à être connues mais leur relation avec la prédisposition au cancer chez le fumeur reste à établir. Le rôle du tabac dans les maladies cardiovasculaires varie aussi en fonction du patrimoine génétique: le risque relatif de développer un infarctus du myocarde dépend à la fois du polymorphisme génétique d'un récepteur d'une glycoprotéine plaquettaire associé à l'existence ou non d'un tabagisme [6]. Une susceptibilité génétique du niveau de la pression artérielle à la prise de sel a été documentée chez I'homme. Expérimentalement, il est clair qu'un facteur alimentaire, un polluant, un toxique exerce un effet différent selon le patrimoine génétique. Ainsi la surcharge en sel dans l'alimentation entraîne une élévation de la pression artérielle chez la souris dont l'un des deux allèles du facteur natriurétique auriculaire a été inactivé, alors même que la pression artérielle ne diffère pas de celle des souris contrôle dans des conditions basales [7].

L'un des grands enjeux de la médecine de demain est d'aboutir à une prévision des risques encourus tant au niveau d'un groupe d'individus que d'une personne donnée. Cependant, prédire la réponse individuelle de chacun à un paramètre précis de I'environnement sera un travail de longue haleine, étant donné le nombre de gènes qui peuvent être impliqués, l'existence de nombreux variants, l'impact modeste que chacun d'entre eux peut exercer sur les paramètres physio-pathologiques. Améliorer la santé de l'homme nécessite plusieurs démarches concomitantes. Bien sûr, l'étude des gènes et de leur fonction, mais tout autant une meilleure connaissance du milieu dans lequel I'homme vit, en tant qu'individu ou société. Cette recherche est par nature pluridisciplinaire. Elle fait intervenir des chercheurs des sciences de la terre et de l'univers, des biologistes, des médecins, des chercheurs des sciences de l'homme et de la société. Elle nécessite un effort d'imagination, la création de nouvelles technologies, une concertation soutenue entre les différentes disciplines scientifiques, une volonté politique perenne du fait de son long terme. Elle est par nature internationale, car l'environnement ne connaît pas de frontière nationale. Il est illusoire de penser que cette recherche aboutira à une société à risque zéro, mais elle permettra d'apporter des éléments de réponse là où existe actuellement le doute

\section{RÉFÉRENCES}

1. Deléage P. Une histoire de l'écologie. Paris: Flammarion, 1991

2. Veyret P, Pech P. L'homme et son environnement. Paris: Presses Universitaires de France, 1997.

3. Crabbe IC, Wahlsten D, Dudek BC. Genetics of mouse behavior: interactions with laboratory environment. Science 1999; 284: 1670-2.

4. Ghani AC. Ferguson NM, Donnelly CA, Anderson RM. Predicted vCJD mortality in Great Britain. Nature 2000; 406 : 583-4.

5. Postel-Vinay $\mathrm{N}$, Corvol $\mathrm{P}$. Le retour $d u$ Docteur Knock. Paris: Odile Jacob, 2000.

6. Mashgegh K, Wuillemin WA, Redondo M, et al. Association of two silent polymorphisms of platelet glycoprotein la/Ila receptor with risk of myocardial infarction: a casecontrol study. Lancet 1999; 353: 351-4.

7 John SWM, Krege JH, Oliver PM, et al. Genetic decreases in atrial natriuretic peptide and salt-sensitive hypertension. Nature $1995 ; 267: 679-81$.

\section{TIRÉS À PART}

P. Corvol. 